\title{
Rigidity of a simple extended lower triangular matrix
}

\author{
Meena Mahajan ${ }^{\text {a }}$ Jayalal Sarma M.N. ${ }^{\text {a }}$ \\ ${ }^{a}$ The Institute of Mathematical Sciences, Chennai 600 113, India.
}

\begin{abstract}
For the all-ones lower triangular matrices, the upper and lower bounds on rigidity are known to match [13]. In this short note, we apply these techniques to the allones extended lower triangular matrices, to obtain upper and lower bounds with a small gap between the two; we show that the rigidity is $\theta\left(\frac{n^{2}}{r}\right)$.
\end{abstract}

Key words: combinatorial problems, computational complexity, matrix rank

For a square matrix over any field, the rigidity function is defined as

$$
R_{M}(r) \stackrel{\text { def }}{=} \inf _{N}\{\operatorname{support}(N): \operatorname{rank}(M+N) \leq r\}
$$

where $\operatorname{support}(N)=\#\{(i, j) \mid N[i, j] \neq 0\}$. The rigidity of a matrix is thus the minimum number of entries that need to be changed to bring down the rank below a given value. A folklore result is that over any field, $R_{M}(r) \leq(n-r)^{2}$. The notion of rigidity was introduced by Valiant [16] and was independently proposed by Grigoriev [6].

The main motivation for studying rigidity is that good lower bounds on rigidity give important complexity-theoretic results in various computational models, such as linear algebraic circuits and communication complexity. An important result in this direction, established by Valiant [16], says that if for some $\epsilon>0$ there exists a $\delta>0$ such that an $n \times n$ matrix $M_{n}$ has rigidity $R_{M_{n}}(\epsilon n) \geq n^{1+\delta}$ over a field $\mathbb{F}$, then the transformation $x \rightarrow M x$ cannot be computed by linear size logarithmic depth linear circuits. See [2] for a survey of this result. Razborov [14] proves that good lower bounds on rigidity over a finite field imply strong separation results in communication complexity: For an explicit infinite sequence of $(0,1)$-matrices $\left\{M_{n}\right\}$ over a finite field $F$, if $R_{M}(r) \geq$

Email addresses: meena@imsc.res.in (Meena Mahajan), jayalal@imsc.res.in (Jayalal Sarma M.N.). 
$\frac{n^{2}}{2^{(\log r)^{o(1)}}}$ for some $r \geq 2^{(\log \log n)^{\omega(1)}}$, then there is an explicit language $L_{M} \notin$ $\mathrm{PH}^{c c}$, where $\mathrm{PH}^{c c}$ is the analog of $\mathrm{PH}$ in the communication complexity setting. See $[3,8]$ for surveys.

However, obtaining explicit bounds on the rigidity of special family of matrices is surprisingly elusive, and thus has received a lot of attention $[4,5,8,9,12,15]$ Recently, Lokam [10] proved the first unconditional super-linear (in fact, quadratic) lower bound for rigidity for an explicit family of matrices (over $\mathbb{C}$ ). However, similar results are not known for $\mathbb{Q}$ or for finite fields $\mathbb{F}_{q}$ for any $q \geq 2$. The rareness of matching, or even close, lower and upper bounds correlates well with the lack of upper bounds on the computational version of rigidity [11]. Due to the difficulty in obtaining non-trivial bounds, the exploration of combinatorial techniques that may lead to such bounds becomes interesting.

A rare case where a closed-form expression has been obtained for rigidity is for the all-ones lower triangular matrices $T_{n}$. By all-ones we mean that any entry permitted to be non-zero is one. That is, $T_{n}$ is the matrix of dimension $n$ with $j \leq i \Longrightarrow T_{n}[i, j]=1, j>i \Longrightarrow T_{n}[i, j]=0$. It is shown in [13] that over any field,

$$
R_{T_{n}}(r)=\frac{(n-r+\Delta)(n+r-\Delta+1)}{2(2 r+1)}
$$

where $n=2 r k+r+k+\Delta$ for $k \geq 0,1 \leq \Delta \leq 2 r+1$.

In this note we consider all-ones extended lower triangular (elt) matrices. In an elt matrix $M$, the first diagonal above the main diagonal can be non-zero, but all other elements above the diagonal must be 0 . (That is, $M[i, j] \neq$ $0 \Longrightarrow j \leq i+1$.) It is worthwhile noting that elt matrices can capture a lot of information: it is known that determinant/permanent computation of elt matrices is as hard as the general case, see [1,7]. An all-ones elt matrix $E L_{n}$ of dimension $n$ is an elt matrix satisfying $j \leq i+1 \Longrightarrow M[i, j]=1$, and has rank $n-1$. Even with this small extension beyond $T_{n}$, we are unable to obtain a closed-form expression for rigidity. However, applying a slight modification of the proof of [13], we show lower and upper bounds differing by an additive factor of roughly $n / r$.

What we find interesting is that though the modification to the matrix family considered in [13] is extremely slight, we are not able to match the lower and upper bounds. Nor does the proof indicate indicate where the slack is: which of the bounds is less likely to be tight. We believe that exploring such combinatorics can help in improving lower bounds.

Theorem 1 Given $n$ and $r$ such that $r \leq n-2$, define the following quantities: $k=\left\lfloor\frac{n-r-1}{2 r+1}\right\rfloor ; \delta=n-r-k(2 r+1) ; \Gamma=\frac{(k+1)}{2}(n-r+\delta) ; \ell=\left\lfloor\frac{n-r}{2 r+1}\right\rfloor$. Now, over any field, 
(1) If $n \leq 3 r$, then $R_{E L_{n}}(r)=n-r-1$.

(2) If $n \geq 3 r+1$, then $\Gamma \leq R_{E L_{n}}(r) \leq \Gamma+\ell-1$.

Asymptotically, $R_{E L_{n}}(r) \in \theta\left(\frac{n^{2}}{r}\right)$.

Our upper bound proof directly mimics that of [13]. Our lower bound proof mimics that of [13] to obtain one bound, and then further tightens it when $n=3 r+1$. A combinatorial argument that can provide a similar tightening at all $n=r+k(2 r+1)$ would completely close the gap between the upper and lower bounds, but we do not see how to obtain this.

Upper Bound: Define $\tau=n-r-(2 r+1) \ell$. We will show that

$$
R_{E L_{n}}(r) \leq \frac{(\ell+1)}{2}(n-r+\tau)+\ell-1
$$

This immediately yields the claimed upper bound when $n \leq 3 r$, since $l=0$ in this case. When $n \geq 3 r+1$, consider two cases:

Case 1: $\ell=k$. Then $\tau=\delta$ and so $\Gamma=\frac{(k+1)}{2}(n-r+\delta)=\frac{(\ell+1)}{2}(n-r+\tau)$.

Case 2 : $\ell=k+1$. Then $\tau=0, \delta=2 r+1$, and $n=2 r \ell+r+\ell=\delta \ell+r$. So

$$
\begin{aligned}
\Gamma & =\frac{(k+1)}{2}(n-r+\delta) \\
& =\frac{(\ell+1)}{2}(n-r+\delta)-\frac{1}{2}(n-r+\delta) \\
& =\frac{(\ell+1)}{2}(n-r+\tau)+\frac{(\ell+1)}{2}(\delta)-\frac{1}{2}(\delta \ell+\delta) \\
& =\frac{(\ell+1)}{2}(n-r+\tau)
\end{aligned}
$$

Thus in either case, the upper bound holds.

Now we establish the upper bound in terms of $\ell$ and $\tau$.

We start with the matrix $E L_{n}$, which has rank $n-1$. In particular, the first $n-1$ rows are linearly independent. Thus to bring the rank down to $r$ or less, at most $r$ of these rows can remain unchanged. We can view the changes as being made sequentially, and track the ranks of matrices along the way, beginning with $n-1$ and ending with $r^{\prime} \leq r$. Since changing a single entry of any matrix changes the rank by at most 1, the optimal way to reduce rank will have $r^{\prime}=r$. Our upper bound assumes that the $r$ linearly independent rows in the final matrix are in fact unchanged rows of $E L_{n}$. (It is possible that a better upper bound exists, that does not use this assumption. But we were unable to derive one, and we think it is unlikely.)

We identify $r$ linearly independent rows $R_{j_{1}}, \ldots R_{j_{r}}$ which we will keep intact, so the rank of the resulting matrix is still at least $r$. We will change each of the other rows to one of these rows by changing some entries. But to minimize 
the number of entries changed, we adopt the following general strategy used in [13] for $T_{n}$. Let $n_{0}$ be the first set of rows which we will explicitly make zero. Similarly, $n_{2 i-1}$ is the number of rows just above $R_{j_{i}}$ which are changed to $R_{j_{i}}$ by changing the appropriate $0 \mathrm{~s}$ to $1 \mathrm{~s}$, and $n_{2 i}$ is the number of rows below the row $R_{j_{i}}$ which are changed to $R_{j_{i}}$ by changing the appropriate 1 s to 0s. Now the total number of changes is a function of these $n_{i}$ 's, as described below, and the natural idea for minimizing the number of changes be to make the contribution of each $n_{i}$ roughly equal. In particular, this evenly spaces out the rows to be preserved. In detail:

$$
\begin{aligned}
\text { \# of changes in } n_{0} \text {-block } & =\sum_{t=1}^{n_{0}}(t+1) & =\frac{n_{0}\left(n_{0}+3\right)}{2} \\
\text { \# of changes in } n_{2 i-1} \text {-block } & =\sum_{t=1}^{n_{2 i-1}} t & =\frac{n_{2 i-1}\left(n_{2 i-1}+1\right)}{2} \\
\text { \# of changes in } n_{2 i} \text {-block } & =\sum_{t=1}^{n_{2 i}} t & \left.=\frac{n_{2 i}\left(n_{2 i}+1\right)}{2}\right) \\
\text { \# of changes in } n_{2 r} \text {-block } & =n_{2 r}-1+\sum_{t=1}^{n_{2 r}-1} t & =\frac{\left(n_{2 r}+2\right)\left(n_{2 r}-1\right)}{2}
\end{aligned}
$$

and we want to minimize the total number of changes.

Intuitively, the optimal choice to achieve this should be to make all the $n_{i}$ 's equal, except $n_{0}$ which should be one less. This is because for each $i \notin\{0, r\}$, some row needs $n_{i}$ changes, and for the extreme blocks the maximum change needed is $n_{0}+1$ and $n_{2 r}-1$ respectively, due to the elt structure. We just try to minimize this maximum change per block. While we cannot show that this strategy is indeed optimal, we use it to obtain our upper bound. When $\tau=2 r$; we set $n_{0}=\ell, n_{i}=\ell+1$ for $i \geq 1$. When $\tau<2 r$, some of the blocks other than $n_{0}$ will also have size $\ell$ rather than $\ell+1$. Thus the last $\tau$ blocks will have size $\ell+1$, and the first $(2 r+1-\tau)$ will be of size $\ell$. Thus,

$$
\begin{aligned}
\text { Total number of changes } & =\frac{\ell(\ell+1)}{2}(2 r+1)+\ell-1+(\ell+1) \tau \\
& =\frac{(\ell+1)}{2}[n-r+\tau]+\ell-1
\end{aligned}
$$

Lower Bound: The lower bound when $n \leq 3 r$ is easy to see: for decreasing the rank of any matrix, at least one entry has to be changed.

The lower bound when $n \geq 3 r+1$ is a little more tricky. In [13], the corresponding lower bound for lower triangular matrices $T_{n}$ is obtained by first showing that if $T_{n}+B_{n}$ has rank bounded by $r$, then some row of $B_{n}$ has at least $k+1$ non-zero entries. Deleting this row and column yields $T_{n-1}+B_{n-1}$ also of rank bounded by $r$. Applying this argument repeatedly, the total number of changes is bounded by a certain sum, yielding the result. Our proof follows the same outline, and differs in essentially two places: (a) Deleting any row $i$ and column $i+1$ of $E L_{n}$ yields $E L_{n-1}$. (b) At $n=3 r+1$ a tighter bound is possible. 
Lemma 2 below shows that some row has lots of changes. Lemma 3 shows that when $n=3 r+1$, at least $2 r+1$ changes are needed. Using these lemmas we can establish the lower bound. When $n \geq 3 r+2$, apply Lemma 2 repeatedly, eliminating one dense row each time, preserving the ELT structure, until $n$ comes down to $3 r+1$. Now Lemma 3 says that $2 r+1$ more changes are necessary. Thus the total number of changes is at least $\delta(k+1)+(2 r+1) k+$ $(2 r+1)(k-1)+\ldots+(2 r+1) 3+(2 r+1) 2+(2 r+1)=\frac{(k+1)}{2}(n-r+\delta)$, giving the lower bound.

We now proceed to state and prove the lemmas.

Note that $k$ and $\delta$ are functions of $n$ and $r$. If we fix $r$ and vary $n$, then $k(2 r+1)+r+1 \leq n \leq k(2 r+1)+3 r+1$. The value of $k$ remains unchanged for $2 r+1$ successive values of $n$, during which $\delta$ ranges over 1 to $2 r+1$.

If $r+2 \leq n \leq 3 r+1$, there is a row with at least 1 change. Now, for a general $n$, assuming that $E L_{n}+B_{n}$ has rank bounded by $r$, repeated applications of the following lemma show that $B_{n}$ has reasonable row-wise density.

Lemma 2 Let $r \leq n-2$, and let $B_{n}$ be a matrix such that $\operatorname{rank}\left(E L_{n}+B_{n}\right) \leq r$. Then some row in $B_{n}$, other than the last row, has at least $(k+1)$ non-zeroes.

Proof: This proof is similar to that in [13]. Assume to the contrary that every row of $B_{n}$ (possibly other than row $n$ ) has fewer non-zeroes than required. Let $A_{n}=E L_{n}+B_{n}$. The idea is to choose a set $S$ of $r+1$ rows which exclude row $n$ (and hence are linearly independent in $E L_{n}$ ), and are linearly dependent in $A_{n}$, and to then show that one of the rows from $S$ in $B_{n}$ has many non-zeroes. We choose $S$ as follows

$$
S=\{k, k+(2 k+1), \ldots, k+r(2 k+1)\}
$$

Since $\operatorname{rank}\left(A_{n}\right) \leq r$, the rows indexed by $S$ are linearly dependent in $A_{n}$; hence for some non-empty subset $S^{\prime}$ of $S$, we have non-zero $\alpha_{j}$ 's satisfying

$$
\sum_{j \in S^{\prime}} \alpha_{j} a_{j}=0 \quad \text { and hence } \quad \sum_{j \in S^{\prime}} \alpha_{j} l_{j}=-\sum_{j \in S^{\prime}} \alpha_{j} b_{j}
$$

Here $a_{j}, l_{j}, b_{j}$ refer to the $j$ th row vectors of $A_{n}, E L_{n}$ and $B_{n}$ respectively. By our assumption, the vector on the right-hand-side RHS has at most $s^{\prime} k$ non-zero entries $\left(s^{\prime}=\left|S^{\prime}\right|\right)$. Exploiting the special structure of the matrix, we show that the left-hand-side LHS has more non-zero terms than the RHS and get a contradiction. Due to the structure of $E L_{n}$, the LHS is of the form $\left(c_{1}, c_{1} \ldots c_{1}, c_{2}, c_{2} \ldots c_{2}, \ldots c_{s^{\prime}} \ldots c_{s^{\prime}}, 0 \ldots 0\right)$. Each $c_{i}$ section is of size at least $2 k+1$, except the $c_{1}$ section, which has size at least $k+1$. Two consecutive sections cannot be zeros since $\alpha_{j} \neq 0$ for all $j$. And the last section necessarily has $c_{s^{\prime}} \neq 0$. 
Case 1: $s^{\prime}=2 t+1$ for some $t$. Now consider the LHS. There are at least $t+1$ blocks of non-zeroes. At most one of these (the first) is of size $k+1$; all the rest have size $2 k+1$. Hence the number of non-zero elements on the LHS is at least $(2 k+1) t+k+1=(2 t+1) k+t+1>s^{\prime} k$.

Case 2: $s^{\prime}=2 t$ with $t \neq 0$. There are at least $t$ blocks of non-zeros. Furthermore, if the first block is a non-zero block, then in fact there must be $t+1$ non-zero blocks. Thus there are at least $t$ blocks of non-zeros of size $2 k+1$. Thus the number of non-zeroes on the LHS is at least $t(2 k+1)>s^{\prime} k$.

Lemma $3 R_{E L_{n}}(r) \geq 2 r+1$ when $n=3 r+1$.

Proof: Suppose not; assume that $2 r$ changes suffice to bring the rank of $E=E L_{3 r+1}$ to $r$ or less. That is, there is a matrix $B$ with at most $2 r$ non-zero entries such that $A=B+E$ has rank $r$ or less. Since there are $3 r+1$ rows, at least $r+1$ of them are left unchanged. These must be linearly dependent to achieve $\operatorname{rank}(A) \leq r$, so they must include rows $n-1$ and $n$ of $E$ (all other rows of $E$ are linearly independent) and exactly $r-1$ other rows.

Let $S$ be the set of indices of preserved rows; $|S|=r+1$ and $\{n-1, n\} \subseteq S$. Let $S^{\prime}=[n] \backslash S$; then $\left|S^{\prime}\right|=2 r$. Each row of $B$ in $S^{\prime}$ has at least one non-zero. But since there are only $2 r$ non-zeroes overall, each row of $B$ in $S^{\prime}$ has, in fact, exactly one non-zero.

For each $i \in S^{\prime}$, row $i$ is dependent on $S$ and on $S \backslash\{n\}$. (With a single change per row, no row cannot be zeroed out.) Let $U=S \backslash\{n\} \cup\{i\}$. Then, as in the proof of Lemma 2, there exists $U^{\prime} \subseteq U: i \in U^{\prime}$, and for each $u \in U^{\prime}, \exists \alpha_{u} \neq 0$ such that

$$
\sum_{u \in U^{\prime}} \alpha_{u} e_{u}=-\sum_{u \in U^{\prime}} \alpha_{u} b_{u}
$$

The RHS has a single non-zero in row $i$ since rows of $B$ from $S$ are zero. The LHS is of the form is of the form $\left(c_{1}, c_{1} \ldots c_{1}, c_{2}, c_{2} \ldots c_{2}, \ldots c_{u^{\prime}} \ldots c_{u^{\prime}}, 0 \ldots 0\right)$ where $c_{u^{\prime}} \neq 0$. To get just one non-zero on the LHS, $c_{u^{\prime}}$ must be a block of size 1 , and all other $c_{j}$ 's must be zero. Thus $\exists k: U^{\prime}=\{k-1, k\}$, and $\alpha_{k}+\alpha_{k-1}=0$. But, we know that $\alpha_{i}$ must be non-zero, since this is the row we are expressing as a combination of rows in $S$. Hence $U^{\prime}$ must be either $\{i-1, i\}$ or $\{i, i+1\}$. Thus, for each row $i \in S^{\prime}$, either row $i-1$ or row $i+1$ is in $S$. So rows in $S$ can be separated by at most 2 rows of $S^{\prime}$. Since rows $n=3 r+1$ and $n-1=3 r$ are in $S$, the 3rd last row of $S$ is at least $3 r-3$, the 4 th last row of $S$ is at least $3 r-6$, and so on; the first row of $S$ is at least row 3. But then row 1 does not have a neighbouring row in $S$, a contradiction. 
We gratefully acknowledge the constructive comments from the anonymous referees, that helped us to improve the readability of this note.

\section{References}

[1] E. Allender, V. Arvind, and M. Mahajan. Arithmetic complexity, Kleene closure, and formal power series. Theory Comput. Syst., 36(4):303-328, 2003.

[2] M. Cheraghchi. On matrix rigidity and the complexity of linear forms. Technical Report TR05-70, ECCC, 2005.

[3] B. Codenotti. Matrix rigidity. Linear Algebra and its Applications, 304(13):181-192, 2000.

[4] B. Codenotti, P. Pudlák, and G. Resta. Some structural properties of low-rank matrices related to computational complexity. Theoretical Computer Science, 235(1):89-107, 2000.

[5] J. Friedman. A Note on matrix rigidity. Combinatorica, 13(2):235-239, 1993.

[6] D. Y. Grigoriev. Using the notions of seperability and independence for proving the lower bounds on the circuit complexity. Notes of the Leningrad branch of the Steklov Mathematical Institute, Nauka, 1976. in Russian.

[7] L. Li. Formal power series: An algebraic approach to the GapP and \#P functions. In Proc. 7th Structure in Complexity Theory Conference, pages 144154, 1992.

[8] S. V. Lokam. Spectral methods for matrix rigidity with applications to sizedepth tradeoffs and communication complexity. In Proc. 36th Annual IEEE Symposium on Foundations of Computer Science, pages 6 - 15, 1995. JCSS 63(3):449-473, 2001.

[9] S. V. Lokam. Note on rigidity of Vandermonde matrices. Theoretical Computer Science, 237(1-2):477-483, 2000.

[10] S. V. Lokam. Quadratic lower bounds on matrix rigidity. In Proc. Theory and Applications of Models of Computation TAMC, LNCS 3959, pages 295-307, 2006.

[11] M. Mahajan and J. Sarma M.N. On the Complexity of Matrix Rank and Rigidity. In Proceedings of 2nd International Computer Science Symposium in Russia (CSR), volume 4649 of Lecture Notes in Computer Science, pages 269280, 2007. Preliminary Techreport appeared as ECCC-TR06-100, Aug 2006.

[12] P. Pudlak and V. Rodl. Some combinatorial-algebraic problems from complexity theory. Discrete Mathematics, 136:253-279, 1994. 
[13] P. Pudlak and Z. Vavrin. Computation of rigidity of order $n^{2} / r$ for one simple matrix. Comment. Nath. Univ. Carolinae., 32(2):213-218, 1991.

[14] A. A. Razborov. On rigid matrices. manuscript in russian, 1989.

[15] D. A. Spielman, M. A. Shokrollahi, and V. Stemann. A remark on Matrix Rigidity. Information Processing Letters, 64(6):283-285, 1997.

[16] L. G. Valiant. Graph theoretic arguments in low-level complexity. In Proc. 6th MFCS, volume 53 of LNCS, pages 162-176. Springer, Berlin, 1977. 